\title{
Claude Charreau (1928-2015)
}

Claude Charreau nous a quittés le 10 juin 2015. Il a marqué durablement l'agronomie, à laquelle il a consacré toute sa carrière, et particulièrement l'agronomie en milieu tropical. Pour rendre hommage à ce grand scientifique à la personnalité très attachante, les Cahiers Agricultures ont recueilli les témoignages de trois personnalités de l'agronomie tropicale qui l'ont bien connu : Jean-François Poulain, René Tourte et Gora Beye.

Jean-François Poulain
Ancien chercheur Cirad et ex-directeur pédagogique du Cnearc,
avec ses compagnons de I'IRAT

Claude Charreau a intégré l'Agro de Paris en 1947, puis l'École supérieure d'agronomie tropicale, où il a opté pour une formation de pédologie. De 1952 à 1957, il a été affecté au bureau des sols à l'Inspection générale de l'agriculture à Dakar. Ce fut un premier contact avec un monde où une douce anarchie se mêlait au respect des traditions et où tant de choses étaient à créer et à imaginer. Ce fut surtout sa découverte du milieu et des sols en zone tropicale sèche, à leur fragilité et à leur sensibilité à l'érosion. Il a intégré le corps de l'ORSTOM (actuel IRD, Institut de recherche pour le développement), détaché dans les services de l'agriculture, puis à l'IRAT (Institut de recherches agronomiques tropicales et des cultures vivrières, intégré à l'actuel Cirad Centre de coopération internationale en recherche agronomique pour le développement).

Claude a rejoint en 1957 le Centre de recherches agronomiques de Bambey, où il est resté jusqu'en 1970. C'est à Bambey que j'ai eu la chance de compléter ma formation grâce à lui pendant une dizaine d'années. En 1974, il a été nommé visiting professor à l'université de Cornell aux États-Unis. Sa carrière internationale l'a alors dirigé vers l'Institut international de recherche sur les cultures des zones tropicales semi-arides (ICRISAT), spécialisé en particulier sur le mil et le sorgho. De 1983 à 1990 , il a été membre de l'ICRISAT Board of Trustees, et il a assuré les mêmes fonctions au Centre international de recherche agricoles sur les zones sèches (ICARDA). Claude a été directeur des programmes Afrique de l'Ouest de l'ICRISAT de 1975 à 1981 , à Dakar. De retour à Nogent, il a été responsable de la coordination des programmes de l'IRAT et a succédé, à sa direction générale, à notre très estimé Francis Bour. De 1985 à 1991, Claude a piloté le passage et les modifications indispensables des activités de l'IRAT vers le Cirad nouvellement institué.

Claude était membre correspondant de l'Académie d'agriculture et Chevalier de la légion d'honneur et de l'Ordre du mérite sénégalais. C'était un homme de contact et d'amitié. Il recherchait toujours les relations avec les autres et souhaitait l'harmonie. Il était choqué par les passe-droits, les arrangements et les compromis. C'était un homme droit et intègre, mais mesuré et animé par le respect des autres. Il était l'auteur ou l'animateur principal d'équipes de rédaction, de publications de qualité axées sur l'amélioration des sols en zones tropicales sèches. Sa fidélité à ses engagements personnels, mais aussi aux objectifs et directives assignés par les organisations pour lesquels il œuvrait, lui ont valu une reconnaissance et une notoriété internationales. Il est en particulier l'artisan principal du rapprochement et de la collaboration avec les organisations internationales et les universités anglophones. Ses amis et camarades de travail, qui ont partagé ses valeurs et accepté de faire équipe avec lui dans son engagement professionnel, social et culturel, n'oublieront pas sa grande capacité à comprendre les attentes de ses interlocuteurs et l'amitié chaleureuse et féconde d'un grand scientifique.

René Tourte

Ancien directeur du Département systèmes agraires du Cirad

Né le 7 novembre 1928 dans une famille de médecins, Claude Charreau avait choisi d'être médecin, non des hommes, mais des sols.

Dès son arrivée à Dakar en 1952, il a été nommé responsable du Bureau des sols de l'Inspection générale de l'agriculture, dont la prime activité était d'élaborer les méthodes et techniques propres à lutter contre l'érosion et ainsi assurer la conservation de ces sols tropicaux réputés fragiles, dont plusieurs auteurs prédisaient les pires dégradations sous l'effet des éléments naturels, mais aussi, et surtout, des pratiques de l'homme et de ses machines. Claude a ainsi ouvré pendant cinq années à la mise en place

doi: 10.1684/agr.2015.0774 


\section{Hommage}

de dispositifs d'observation, de mesure et de contrôle de l'érosion en différentes conditions naturelles ou modifiées par l'homme : " parcelles d'érosion ", " bassins-versants ", etc.

L'étape majeure suivante, celle de la protection et de la bonification de ces sols menacés, Claude l'a abordée en 1957 au Centre de recherches agronomiques de Bambey. Il y a succédé au grand agrologue Serge Bouyer et a pris en charge l'étude et l'amélioration des sols, plus ou moins appauvris ou dégradés par l'exploitation (cultures, élevages). Plus largement, c'est au complexe eau-sol-plante, au milieu pédoclimatique, que Claude a consacré ses efforts les treize années suivantes, avec son équipe de chercheurs, ingénieurs, techniciens, qui se sont tous loués de ses hautes compétences, de sa large expérience, de ses qualités de chef et de sa constante urbanité.

Outre quelques prospections et cartographies pédologiques, locales ou régionales, Claude a alors poursuivi des études portant sur la dynamique des eaux et des éléments minéraux dans le sol, grâce notamment à des dispositifs élaborés du type cases lysimétriques, sondes à neutrons, marqueurs isotopiques, etc. Ces études ont permis une conduite de plus en plus maîtrisée de l'amendement et de la fertilisation des sols tropicaux, notamment des savanes. De plus, grâce à des dispositifs expérimentaux pluriannuels, Claude a mis un accent particulier et novateur sur l'étude de l'évolution des sols sous culture dans leurs caractéristiques physiques, chimiques, hydriques et sur leurs potentialités. En liaison avec les agronomes, il a alors proposé des techniques de redressement et d'entretien de la fertilité des sols aux pas de temps des rotations et successions culturales.

En 1970, Claude, riche d'expérience et d'une exceptionnelle connaissance des sols tropicaux, a rejoint le siège de l'IRAT à Nogent-sur-Marne. En 1974, il a dispensé à la prestigieuse Université américaine de Cornell une série de conférences magistrales sur les sols des régions semi-arides de l'Afrique de l'Ouest et leur mise en valeur. Puis, il a été sollicité par l'ICRISAT pour diriger son programme coopératif en Afrique de l'Ouest. Cette nouvelle période africaine lui a permis d'affirmer un peu plus ses liens profonds avec cette Afrique qu'il a aimée et à laquelle il a sans doute consacré le meilleur de sa vie professionnelle et familiale. Puis a sonné le retour définitif en métropole, où Claude a dirigé jusqu'à sa retraite l'IRAT, qui employait près de 400 personnes et intervenait dans quelque 25 pays.

Tous les amis de Claude Charreau savent quelle insigne contribution à la recherche agronomique française et internationale a été la sienne et quelle cruelle perte cause son départ.

\section{Gora Beye \\ Ancien chercheur au CNRA de Bambey}

J'ai connu Claude Charreau en octobre 1965 à mon recrutement à l'IRAT et affectation au CNRA (Centre national de recherches agronomiques) de Bambey. Jeune pédologue, fraîchement sorti de l'ORSTOM (actuel IRD) et formaté pour la pédologie classique (cartographie, pédogénèse, etc.), il m’a pris en charge et intégré facilement dans le Groupe des sols, en me faisant comprendre gentiment que les pédologues au CNRA étaient plutôt des agro-pédologues soucieux essentiellement de mettre la plante dans les meilleures conditions de croissance et de productivité. Il m'a affecté comme assistant au chef d'équipe Mamadou Diallo, dit Diallo Dubois, en référence à un autre précurseur de l'étude des sols sénégalais. Sous la direction de Claude Charreau, j'eus la tâche d'abord de travailler sur des sujets ponctuels : caractérisation des sols de rizières de l'enquête rizicole en Casamance, département d'Oussouye, cartographie de la station de Richard Toll (fleuve Sénégal), diagnostic des cas d'hétérogénéité des parcelles de canne à sucre à Richard Toll, cartographie des Papem (points d'appui pour l'expérimentation multilocale). Il m'orienta par la suite sur l'étude des sols salés, d'abord sur l'évolution de la salinité dans la Cuvette de Boundoum-Ouest dans le delta du fleuve Sénégal, et, après mon affectation à Djibélor (Casamance), sur des sols sulfatés acides et salés. En fait, il s'agissait des domaines qui le préoccupaient déjà avec les travaux qu'il avait engagés dans la vallée du Bao Bolon dans le Sine Saloum. Si je suis devenu un passionné des sols salés et sulfatés acides, c'est grâce à lui. Mais les domaines d'intervention de Claude Charreau dans l'étude des sols embrassent tous les domaines : la fertilité, la conservation, le travail du sol, etc. Sur ce dernier point, il mena une bataille pour montrer l'utilité du travail des sols légers, en particulier au Sénégal et en Afrique tropicale sèche, contrairement aux recommandations des tenants du zéro tillage et du mulching, véhiculées par les chercheurs en zones humides de l'IITA (International Institute of Tropical Agriculture) d'Ibadan, au Nigéria.

Monsieur Claude Charreau a contribué très grandement au développement de la recherche agronomique sénégalaise et ouestafricaine. Je garde de lui le souvenir d'un homme gentil, d'une extrême courtoisie, accueillant, pédagogue, un meneur d'équipe. Il aimait beaucoup le Sénégal, son pays d'adoption. 